\title{
DATOS ACERCA DE LA VEGETACIÓN BRIOFÍTICA TERRÍCOLA EN SALAMANCA
}

\author{
María Jesús ELíAS RIVAS \& José Luis RUPIDERA GIRALDO
}

RESUMEN: Se estudian diversas comunidades briofíticas terrícolas presentes en la provincia de Salamanca, comentándose diversos aspectos sinecológicos, sinfisionómicos, sintaxonómicos y sincorológicos de las mismas.

Palabra clave: Briófitos, vegetación, terrícola, Salamanca.

SUMMARY: This is a study of the terricolous bryophytic vegetation in the Salamanca province, with some comments on synecologic, symphysionomic, syntaxonomic and synchorologic aspects.

Key words: Bryophytic, vegetation, terricolous, Salamanca.

\section{INTRODUCCIÓN}

Las comunidades briofíticas terrícolas, en su mayor parte basófilas, xerófilas y ricas en hepáticas talosas, distribuidas en zonas mediterráneas y submediterráneas, se incluían hasta ahora en la alianza Grimaldion fragantis. En concordancia con este tratamiento sintaxonómico, uno de nosotros (Elías Rivas, 1987:204), incluyó en dicha alianza la vegetación terrícola presente en varios emplazamientos del valle del río Alagón, fuertemente insolados y de neto carácter mediterráneo.

Posteriormente Ros \& Guerra (1987: 522-524) propusieron un nuevo status sintaxonómico, considerando que el comportamiento ecológico de Mannia fragans (Balbis) Frye \& Clark en Centroeuropa es netamente distinto al que presenta en zonas meridionales: dado que en estas comunidades mediterráneas no aparece ninguna de las características de dicha alianza, englobaron las mismas en un nuevo sintaxon, Mannion androgynae, basándose en la nueva asociación Riccio nigrellae-Oxymitretum paleaceae; se trata de una alianza vicariante mediterránea de Grimaldion .

\section{BIOGEOGRAFÍA Y BIOCLIMATOLOGÍA}

En el solar administrativamente salmantino cabe reconocer nítidamente 4 pisos bioclimáticos: meso-, supra-, oro-, y crioromediterráneo. En el primero están incluidos los subsectores Ribaduriense (Carpeto-Ibérico-Leonesa) y Hurdano-Zezerense (Luso- 
Extremadurense), pues en ambas unidades biogeográficas los valores del $\mathrm{I}_{\mathrm{t}}$ oscilan entre los límites establecidos por Rivas-Martínez (1987:37) para poderlas calificar de mesomediterráneas. En la primera unidad se encuadran las áreas occidentales de menor altitud (Arribes del Duero), de ombroclima seco, mientras que a la segunda pertenece el valle del río Alagón con ombroclima húmedo.

Es en estas áreas mesomediterráneas donde hemos detectado la presencia de comunidades terrícolas que creemos representan la vegetación de Riccio-Oxymitretum en el CW español.

\section{DESCRIPCIÓN DE LAS COMUNIDADES}

\section{1.- Riccio nigrellae-Oxymitretum paleaceae}

Sinecología. Se desarrolla sobre suelos de carácter moderadamente ácidos -pH de 6 a 6.5-, colonizando fisuras y rellanos rocosos; en las zonas estudiadas se han encontrado fundamentalmente sobre litosoles y regosoles eútricos, formados bien sobre cornubianitas -valle del Alagón- bien sobre esquistos pizarrosos -arribes del Duero-.

Como anteriormente poníamos de manifiesto, y a tenor de nuestros estudios, en la provincia de Salamanca dichas fitocenosis se presentan exclusivamente en el piso mesomediterráneo cuyo ombroclima oscila de seco a húmedo.

Sinfisionomía y estructura florística. El predominio constante de hepáticas talosas -algunas de tamaño considerable- determina la fisionomía de esta comunidad.

En estas zonas centro-occidentales están representados prácticamente todos los táxones indicados como característicos por Ros \& Guerra (loc. cit.) y con un neto dominio de Oxymitra paleacea, Mannia androgyna y Riccia nigrella; no hemos detectado la presencia de Riccia gougetiana, que parece estar sustituida por R. ciliifera, asimismo bastante abundante. Cabe destacar además la presencia, siquiera puntual, de otro elemento típicamente mediterráneo: Corsinia coriandrina .

Sintaxonomía y Sincorología. Esta comunidad fue descrita por Ros \& Guerra (loc. cit.) en el seno de la nueva alianza Mannion androgynae, sintaxon vicariante mediterráneo del Grimaldion fragantis centroeuropeo.

Queda patente la abundancia y predominio de elementos de dicha alianza en las comunidades estudiadas en la provincia de Salamanca (ver Tabla I).

Según los datos actuales disponibles, dicha asociación presentaría una distribución circunmediterránea. En cuanto a la Península Ibérica se conoce de zonas litorales térmicas y ahora la señalamos para el centro-oeste español, donde aparece bien representada. 


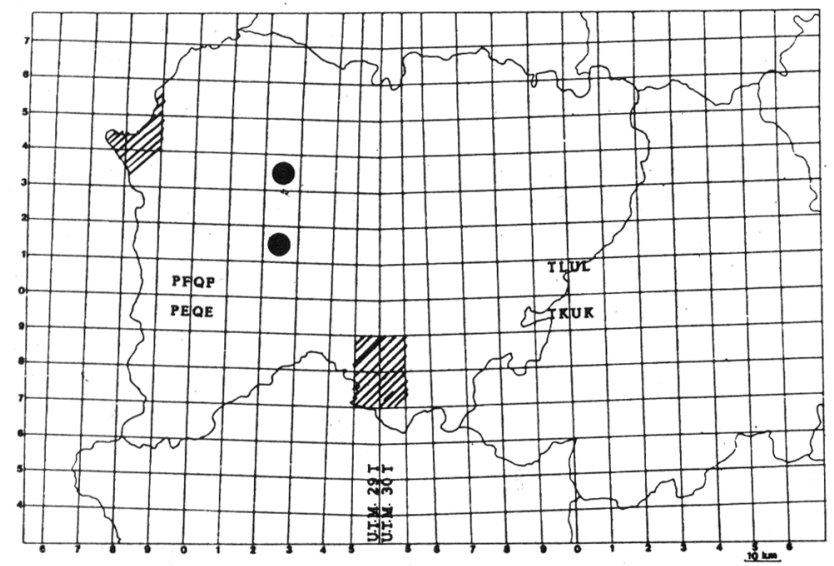

Fig. 1.- Distribución de la asociación Riccio-Oxymitretum ( ///// ) y de las comunidades presididas por Exormotheca bullosa $(\bullet)$ en la provincia de Salamanca.

\section{2.- Comunidades con Exormotheca bullosa}

En algunas áreas de la penillanura salmantina -Sancti Spiritus, Villares de Yeltes, fig.1- incluídas en el sector Salmantino (Carpetano-Ibérico-Leonesa), correspondientes al piso supramediterráneo y de ombroclima seco a subhúmedo, hemos detectado la presencia de unas particulares comunidades presididas por la hepática mesoxerófila Exormotheca bullosa (Tabla II).

Se presentan sobre suelos de gran desarrollo, pedregosos y esqueléticos, con un horizonte superior de textura arenosa, formados sobre depósitos de «rañas», y que parecen corresponder a luvisoles crómicos.

Colonizan suelos desnudos en pequeñas áreas que no albergan vegetación cormofítica de Cicendion, en el seno de robledales del Genisto falcatae-Quercetum pyrenaicae, y representan una vegetación pionera de suelos temporalmente inundados por aguas dulces (fig.2).

Creemos que su inclusión en el orden Barbuletalia unguiculatae resulta evidente. Así mismo, y con los conocimientos actuales sobre dicho orden, parece adecuado encuadrar dichas comunidades en la alianza Phascion cuspidatae, la única que logra una óptima representación en climas mediterráneos de ombroclima no árido o semiárido.

Si bien estas comunidades parecen estar bien definidas tanto florística como ecológicamente, no nos parece oportuno en estos momentos la propuesta de un nuevo sintaxon para las mismas, dada la peculiar distribución de Exormotheca bullosa en nuestra Península (cf. Puche \& al., 1987: 247), y puesto que no conocemos bien su ecología en el resto de las localidades donde está presente. 
Tabla I

Riccio nigrellae-Oxymitretum paleaceae

(Mannion androgynae, Barbuletalia unguiculatae, Barbuletea unguiculatae)

Número de orden

Altitud (m.s.n.m.)

Area en $\mathrm{dm}^{2}$

Cobertura $(\%)$

Inclinación $(\stackrel{\circ}{)}$

Orientación

Número de especies

$\begin{array}{rr}1 & 2 \\ 175 & 625 \\ 7 & 5 \\ 90 & 80 \\ 25 & 5 \\ \text { SO } & \text { SE } \\ 13 & 14\end{array}$

$\begin{array}{rrr}3 & 4 & 5 \\ 600 & 610 & 150 \\ 5 & 6 & 8 \\ 40 & 40 & 80 \\ 10 & 10 & 15 \\ \mathrm{~N} & \mathrm{~N} & \mathrm{SO} \\ 10 & 8 & 12\end{array}$

Características de asociación y alianza:

Oxymitra paleacea

Targionia hypophylla

Mannia androgyna

Riccia ciliifera

Riccia nigrella

Targionia lorbeeriana

Riccia trichocarpa

Corsinia coriandrina

Características de unidades superiores:

Fossombronia angulosa

Bryum bicolor

Bryum gemmilucens

Weissia controversa

Riccia sorocarpa

Riccia crozalsii

Compañeras:

Bryum argenteum

Scapania compacta

Bartramia stricta

Pleuridium acuminatum

$\begin{array}{lllll}3 & 1 & 2 & 1 & 2 \\ 2 & + & 1 & + & 2 \\ 2 & 3 & - & 2 & 3 \\ 1 & 1 & 1 & - & + \\ 1 & 2 & - & - & 1 \\ 2 & - & - & - & 2 \\ - & + & - & - & - \\ + & - & - & - & -\end{array}$

Localidades. SALAMANCA: 1, La Fregeneda, muelle de Vegaterrón, Arribes del Duero, 29TPF7444. 2, Entre Valero y Miranda, valle del Alagón, 30TTK5190. 3, Montemayor del Río, valle de Montemayor, 30TTK5170. 4, Valdelageve, 30TTK4978. 5, La Fregeneda, muelle de Vegaterrón, Arribes del Duero, 29TPF7444.

\section{ESQUEMA SINTAXONOMICO}

Barbuletea unguiculatae von Hübschmann 1967

Barbuletalia unguiculatae von Hübschmann 1967

Mannion androgynae Ros \& Guerra 1987

Riccio nigrellae-Oxymitretum paleaceae Ros \& Guerra 1987

Phascion cuspidatae Walheim 1944

Asociación (?) Comunidades con Exormotheca bullosa 
Tabla II

Comunidades presididas por Exormotheca bullosa

(Phascion cuspidatae, Barbuletalia unguiculatae, Barbuletea unguiculatae)

Número de orden

Altitud (m.s.n.m.)

Area en $\mathrm{dm}^{2}$

Cobertura (\%)

Número de especies

Exormotheca bullosa

Enthostodon fascicularis

Riccia sorocarpa

Barbula unguiculata

Encalypta vulgaris

Gongylanthus ericetorum

Fossombronia pusilla

Riccia macrocarpa

Bryum bicolor

Bryum argenteum

Pleuridium acuminatum

Cephaloziella divaricata

$\begin{array}{rr}1 & 2 \\ 800 & 775 \\ 6 & 7 \\ 55 & 40 \\ 12 & 8\end{array}$

$\begin{array}{ll}3 & 2 \\ 1 & 1 \\ 1 & 1 \\ + & + \\ + & + \\ + & + \\ + & - \\ + & - \\ + & - \\ + & - \\ + & + \\ - & \end{array}$

Localidades: SALAMANCA: 1, Sancti-Spiritus, 29TQF2313. 2, Entre Cubo y Pozo de Hinojos, 29TQF2234.

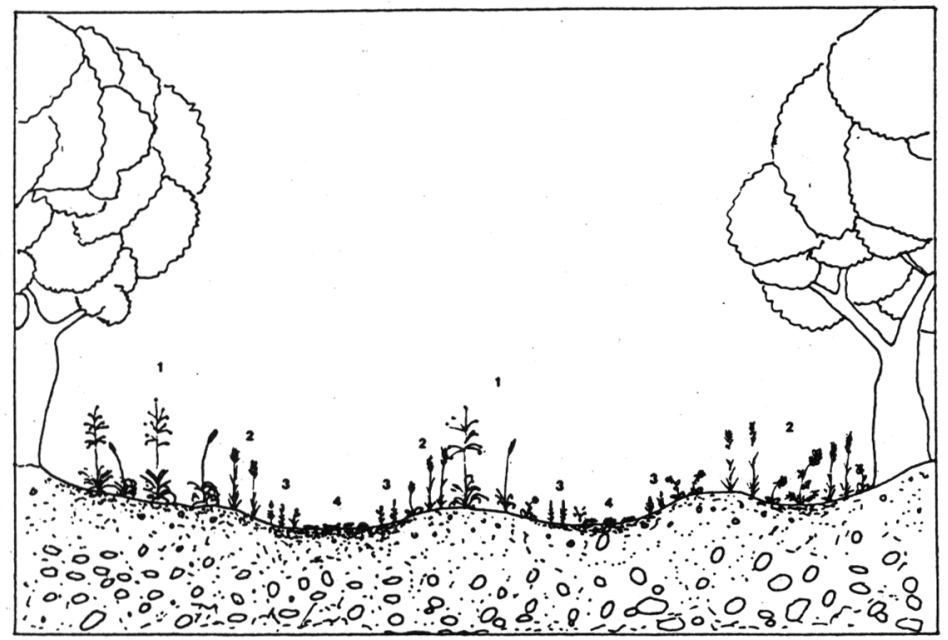

Fig. 2.- Situación topográfica de las comunidades con Exormotheca bullosa: 1. Agrostion castellanae. 2. Cynosurion cristati. 3. Cicendion. 4. Comunidades pioneras con Exormotheca 


\section{BIBLIOGRAFÍA}

ELIAS RIVAS, M J. -1987-Estudio de la flora briológica de las sierras de Béjar y Peña de Francia. Tesis Doctoral inéd. Facultad de Biología. Salamanca.

PUCHE, F., A. CASAÑ \& J. J. HERRERO-BORGOÑON -1987- El género Exormotheca Mitt. (Hepaticophytina) en la Península Ibérica. Acta Bot Malacitana 12: 246-248.

RIVAS-MARTINEZ, S. -1987- Nociones sobre Fitosociología, Biogeografía y Bioclimatología in PEINADO, M. \& S. RIVAS-MARTINEZ (eds.), La vegetación de España: 19-45. Ser. Publ. Alcalá de Henares.

ROS, R. M. \& J. GUERRA -1987- Vegetación briofítica terrícola de la región de Murcia (sureste de España). Phytocoenologia 15: 505-567.

(Aceptado para su publicación en Junio de 1.990)

Dirección de los autores: Departamento de Biología Vegetal (Botánica). Facultad de Biología. 37008 Salamanca. 